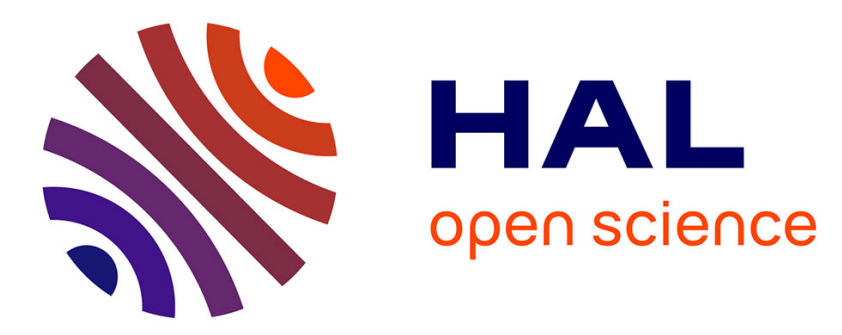

\title{
Can phase masks extend depth-of-field in localization microscopy?
}

Olivier Lévêque, Caroline Kulcsár, Hervé Sauer, Antony Lee, Pierre Bon, Laurent Cognet, François Goudail

\section{To cite this version:}

Olivier Lévêque, Caroline Kulcsár, Hervé Sauer, Antony Lee, Pierre Bon, et al.. Can phase masks extend depth-of-field in localization microscopy?. Unconventional Optical Imaging II, International Society for Optics and Photonics, Apr 2020, Online Only, France. pp.50-58, 10.1117/12.2558426 . hal-03025685

\section{HAL Id: hal-03025685 \\ https://hal.science/hal-03025685}

Submitted on 26 Nov 2020

HAL is a multi-disciplinary open access archive for the deposit and dissemination of scientific research documents, whether they are published or not. The documents may come from teaching and research institutions in France or abroad, or from public or private research centers.
L'archive ouverte pluridisciplinaire HAL, est destinée au dépôt et à la diffusion de documents scientifiques de niveau recherche, publiés ou non, émanant des établissements d'enseignement et de recherche français ou étrangers, des laboratoires publics ou privés. 


\title{
Can phase masks extend depth-of-field in localization microscopy?
}

\author{
Olivier Lévêque ${ }^{\mathrm{a}}$, Caroline Kulcsár ${ }^{\mathrm{a}}$, Hervé Sauer ${ }^{\mathrm{a}}$, Antony Lee ${ }^{\mathrm{b}, \mathrm{c}}$, Pierre Bon ${ }^{\mathrm{b}, \mathrm{c}}$, Laurent \\ Cognet $^{\mathrm{b}, \mathrm{c}}$, and François Goudail ${ }^{\mathrm{a}}$ \\ aUniversité Paris-Saclay, Institut d'Optique Graduate School, CNRS, Laboratoire Charles \\ Fabry, 91127, Palaiseau, France. \\ bUniversité de Bordeaux, Laboratoire Photonique Numérique et Nanosciences, UMR 5298, \\ 33400, Talence, France \\ 'Institut d'Optique \& CNRS, LP2N UMR 5298, 33400, Talence, France
}

\begin{abstract}
In localization microscopy, the position of isolated fluorescent emitters are estimated with a resolution better than the diffraction limit. In order to image thick samples, which are common in biological applications, there is considerable interest in extending the depth-of-field of such microscopes in order to make their accuracy as invariant as possible to defocus. For that purpose, we propose to optimize annular binary phase masks placed in the pupil of the microscope in order to generate a point spread function for which the localization accuracy is almost invariant along the optical axis. The optimization criterion is defined as the localization accuracy in the plane expressed in terms of the Cramér-Rao bound. We show that the optimal masks significantly increase the depth-of-field of single-molecule imaging techniques relatively to an usual microscope objective.
\end{abstract}

Keywords: binary phase masks, depth-of-field extension, single-molecule localization microscopy, co-designed optical system, wavefront coding, image processing, optimization

(C) 2020 Society of Photo-Optical Instrumentation Engineers (SPIE). One print or electronic copy may be made for personal use only. Systematic reproduction and distribution, duplication of any material in this paper for a fee or for commercial purposes, or modification of the content of the paper are prohibited. DOI: $10.1117 / 12.2558426$

\section{INTRODUCTION}

Single-molecule localization microscopy is used to observe biological subcellular structures. This super-resolution imaging technique makes it possible to estimate very accurately the position of an isolated fluorescent emitter in the field of the camera beyond the diffraction limit. ${ }^{1,2}$ A lot of work has been devoted to determine the precision limit of this technique. ${ }^{3-5}$ Globally, the fundamental limit on localization standard deviation depends on the numerical aperture (NA) of the microscope and on the signal-to-noise ratio. ${ }^{5}$ Most of these works have assumed that the microscope is perfectly focused on the observed object.

Over the last decades, various biological applications have focused on imaging thicker samples in which the objects of interest are generally not confined within the two dimensions of the focus plane. Several approaches have therefore been designed to extend the super-localization concept to the third dimension. ${ }^{6}$ These new approaches make it possible to estimate the 3D position of the fluorescent emitters in the natural depth-of-field (DoF) of the high NA objectives used in single-molecule microscopy. In order to further extend the DoF in 3D localization microscopy, sophisticated point spread function (PSF) engineering designs using pupil wavefront coding have been studied but they all inherently require significant photon spread. ${ }^{7-9}$ This leads to considerably reduce the localization performance in the low signal conditions encountered for instance in fast single particle tracking or deep tissue imaging. Another approach consists in extending the DoF by making the PSF of the

Further author information: (Send correspondence to Olivier Lévêque)

Olivier Lévêque: E-mail: olivier.leveque@institutoptique.fr, Telephone: +33 (0)1 64533256 
microscope invariant along the imaging axis to generate volumetric images consisting of $2 \mathrm{D}$ projections of the 3D imaging volume. In this work, we present a methodology to apply this optimization concept not to the shape of the microscope PSF but directly to its localization performance, which is the real parameter of interest.

A similar issue is encountered in classical imaging systems where the DoF can be improved by placing a phase mask in the pupil of the objective and by restoring the image with an appropriate deconvolution filter. ${ }^{10-12}$ The phase function of the mask can be optimized to maximize the quality of the deconvolved image. ${ }^{11,13-15}$ This approach consisting in optimizing an imaging system while taking into account the image formation model, the properties of the optical system and the method of information extraction is called "co-design". 16, 17 Our purpose is to apply this approach to DoF enhancement of localization microscopy. The optimization criterion is different from that used in classical imaging since it is needed to optimize a location accuracy instead of a restored image quality, leading to possibly different optimal masks.

This present work proposes to explore two perspectives described in our previous work. ${ }^{18}$ The first one is to characterize DoF-enhancing phase masks when the measurements are corrupted by signal-dependent photon noise, which is a more realistic model than additive Gaussian noise. The second one is to propose different optimization criteria based on the Cramér-Rao bound (CRB) to extend DoF of localization microscopy.

This paper is organized as follows. Section 2 describes the 2D localization problem and the loss of localization accuracy when the observed particle is out-of-focus. We introduce the Fisher information matrix and remind the concept of the fundamental limit of localization accuracy based on the CRB. In section 3, we present a co-design approach to improve the localization accuracy of defocused imaging systems and introduce the annular binary phase masks that will be used for that purpose. In section 4, we optimize these masks, present and discuss their performance. Conclusions and perspectives are drawn in section 5 .

\section{OUT-OF-FOCUS LOCALIZATION ACCURACY}

Within thick samples, the fluorescent particles of interest may be located outside the best focus range of the imaging system. In this case, the PSF of the emitter varies significantly as a function of its axial position. In this section, we describe the 2D localization problem and the loss of localization accuracy when the observed fluorescent particle is out-of-focus. We introduce the Fisher information matrix and recall the concept of the fundamental limit of localization accuracy based on the CRB.

\subsection{Optical model for defocus}

In the Fresnel approximation, the generalized pupil phase function corresponding to an out-of-focus object is

$$
\Phi(r)=\frac{2 \pi \psi r^{2}}{\lambda}, \quad r \in[0,1]
$$

where $r$ is the normalized radial distance in the polar coordinates of the exit pupil, $\lambda$ is the wavelength of the collected light and $\psi$ is the wavefront error at the edge of the pupil considered as the traditional parameter for quantifying defocus. ${ }^{19}$ This parameter (an optical path difference) is defined as

$$
\psi=\frac{\mathrm{NA}^{2} \Delta z}{2 n}
$$

where NA denotes the object numerical aperture of the imaging system; $\Delta z$ is the longitudinal distance between the observed particle and the nominal in-focus plane in the object space; $n$ is the refractive index of the immersion object space.

The image of the emitter in the sensor plane is the translated PSF of the system:

$$
f^{\psi, \boldsymbol{\theta}_{0}}(x, y)=f^{\psi}\left(x-M x_{\mathrm{p}}, y-M y_{\mathrm{p}}\right)
$$

where $M$ is the lateral magnification of the imaging setup, and $f^{\psi}(x, y)$ is the $2 \mathrm{D}$ spatial distribution of irradiance in the image space when the single-molecule is located at $\boldsymbol{\theta}_{0}=\left(x_{\mathrm{p}}, y_{\mathrm{p}}\right)^{\mathrm{t}}$ in the object plane. The superscript $\mathrm{t}$ denotes transposition. The $2 \mathrm{D}$ spatial distribution of irradiance is normalized so that its integral is equal to 
(a) $\psi=0$

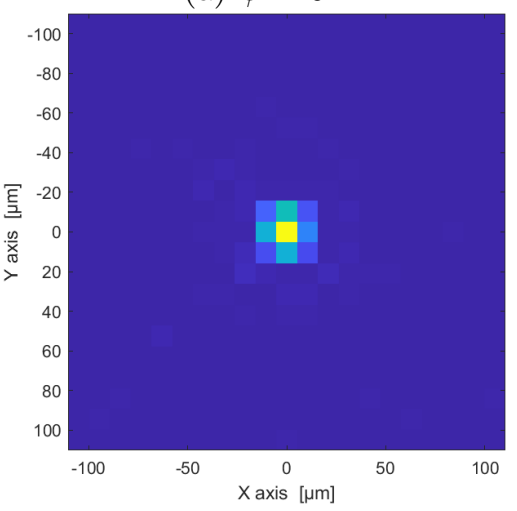

(b) $\psi= \pm 0.5 \lambda$

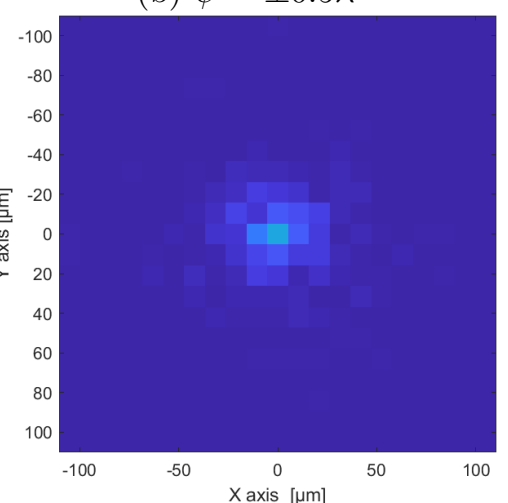

(c) $\psi= \pm 1 \lambda$

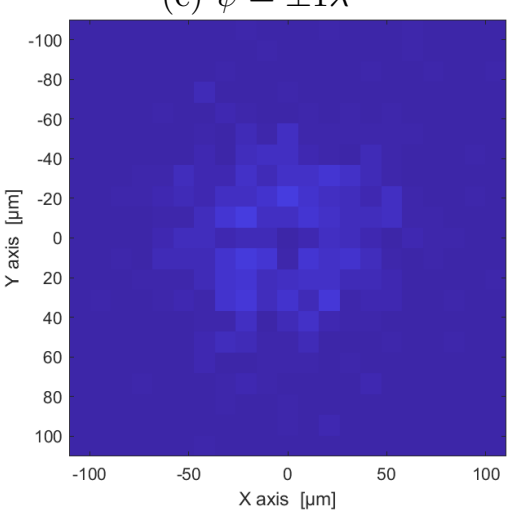

Figure 1. Particle image simulations $s_{i j}$ of an optical localization microscope such as (a) $\psi=0$, (b) $\psi= \pm 0.5 \lambda$ and (c) $\psi= \pm 1 \lambda$. Simulation parameters: $\boldsymbol{\theta}_{0}=(0,0)^{\mathrm{t}} \mu \mathrm{m}, N_{0}=500$ photo-electrons, $\Delta_{x y}=10 \mu \mathrm{m}, 2 K+1=21$ pixels, $\mathrm{NA}=1.3$ (object numerical aperture), $\lambda=700 \mathrm{~nm}$ (wavelength), and $M=60$ (magnification).

1 (i.e. $\iint f^{\psi, \boldsymbol{\theta}_{0}}(x, y) \mathrm{d} x \mathrm{~d} y=1$ ) and is proportional to the squared modulus of the Fourier transform of the generalized pupil function $\exp [i \Phi(r)]$, if $r<1,0$ otherwise with $\Phi(r)$ defined in Eq. (1).

We assume that we observe a $2 \mathrm{D}$ pixelized square image of a single-molecule with a width of $2 K+1$ pixels. To take into account the signal-dependent photon noise, we define $s_{i j}$, the number of photo-electrons observed at pixel $(i, j) \in \mathbb{N}^{2}$ with $|i|$ and $|j| \leq K$, as a Poisson distributed random variable of mean

$$
\mu_{i j}^{\psi, \boldsymbol{\theta}_{0}}=N_{0} \int_{\left(i-\frac{1}{2}\right) \Delta_{x y}}^{\left(i+\frac{1}{2}\right) \Delta_{x y}} \int_{\left(j-\frac{1}{2}\right) \Delta_{x y}}^{\left(j+\frac{1}{2}\right) \Delta_{x y}} f^{\psi, \boldsymbol{\theta}_{0}}(x, y) \mathrm{d} x \mathrm{~d} y
$$

where $N_{0}$ denotes the total number of photo-electrons expected in the whole image and $\Delta_{x y}$ denotes the width of the square pixels.

The $\psi$-dependence of the PSF is illustrated in Fig. 1, where we present 3 particle image simulations obtained for 3 different defocus parameters: $\psi=\{0,0.5 \lambda, 1 \lambda\}$. When $\psi=0$, the object is in focus and the PSF is described by the classical Airy pattern whose size is defined by the object NA of the microscope objective. When $\psi$ increases, the PSF progressively gets wider.

Due to the circular symmetry of the PSF, another way to illustrate its $\psi$-dependence is presented in Fig. 2.a. We have indeed represented the variation of the PSF profile along the $x$-axis as a function of the defocus parameter $\psi$ for an example of microscope configuration. Only the positive values of $\psi$ are illustrated because PSF is identical for $\psi$ and $-\psi$ (i.e. on either side of the focus point). We note that as defocus increases, the PSF spreads out and its central lobe gets fainter.

We therefore perceive that the more $\psi$ increases, the more difficult it is to accurately estimate the position of an emitter because the PSF deteriorates.

\subsection{Fundamental localization accuracy limit}

To quantify the loss of location accuracy with defocus, we introduce the well known concept of fundamental limit of localization accuracy. ${ }^{5}$ This limit can be obtained from the Fisher information matrix defined by

$$
\underline{\boldsymbol{I}}\left(\boldsymbol{\theta}_{0}\right)=\sum_{i=-K}^{K} \sum_{j=-K}^{K} \mathrm{E}\left[\left(\left.\frac{\partial \ln p\left(s_{i j} \mid \mu_{i j}^{\psi, \boldsymbol{\theta}}\right)}{\partial \boldsymbol{\theta}}\right|_{\boldsymbol{\theta}=\boldsymbol{\theta}_{0}}\right)\left(\left.\frac{\partial \ln p\left(s_{i j} \mid \mu_{i j}^{\psi, \boldsymbol{\theta}}\right)}{\partial \boldsymbol{\theta}}\right|_{\boldsymbol{\theta}=\boldsymbol{\theta}_{0}}\right)^{\mathrm{t}}\right]
$$

where the symbol E[.] denotes the mathematical expectation operation and $p\left(s_{i j} \mid \mu_{i j}^{\psi, \boldsymbol{\theta}_{0}}\right)$ is the probability of measuring $s_{i j}$ photo-electrons at pixel $(i, j)$, knowing the average number of expected photo-electrons $\mu_{i j}^{\psi, \boldsymbol{\theta}_{0}}$. 
(a)

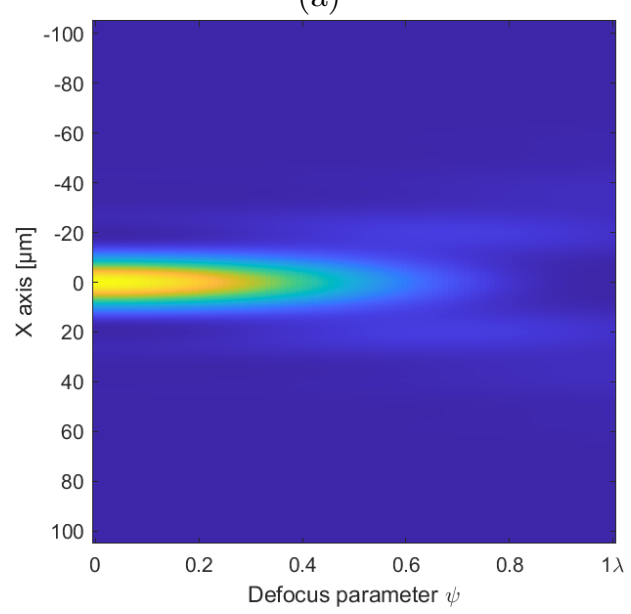

(b)

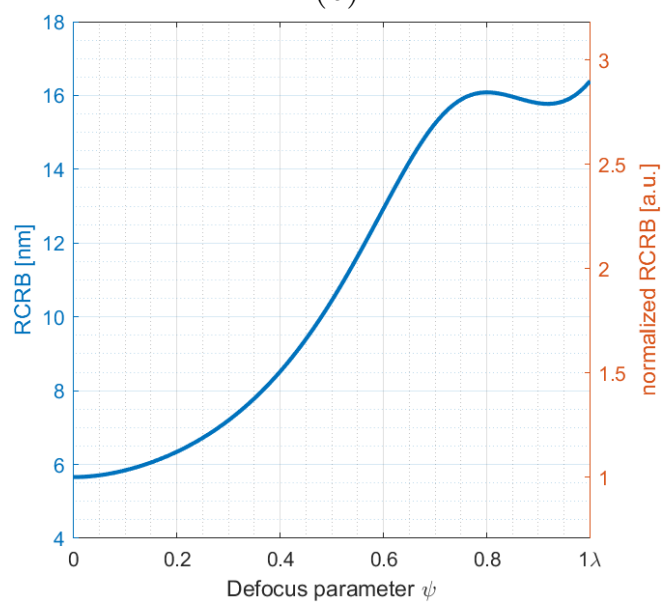

Figure 2. Evolutions of (a) the PSF profile along $x$-axis and (b) the limit of the localization accuracy, denoted RCRB, as a function of the defocus parameter for an aberration-free microscope objective. Simulation parameters: $\boldsymbol{\theta}_{0}=(0,0)^{\mathrm{t}} \mu \mathrm{m}$, $N_{0}=500$ photo-electrons, $\Delta_{x y}=10 \mu \mathrm{m}, 2 K+1=21$ pixels, NA $=1.3$ (object numerical aperture), $\lambda=700 \mathrm{~nm}$ (wavelength), and $M=60$ (magnification).

This probability is defined as

$$
p\left(s_{i j} \mid \mu_{i j}^{\psi, \boldsymbol{\theta}_{0}}\right)=\frac{\left(\mu_{i j}^{\psi, \boldsymbol{\theta}_{0}}\right)^{s_{i j}} \exp \left(-\mu_{i j}^{\psi, \boldsymbol{\theta}_{0}}\right)}{\left(s_{i j}\right) !}
$$

where the symbol (.)! denotes the factorial function.

Due to the circular symmetry of the PSF $f^{\psi, \boldsymbol{\theta}_{0}}(x, y)$ for any value of $\psi$, the off-diagonal terms of the Fisher information matrix are zero and the square root of Cramér-Rao bound, denoted RCRB, is the square root of the inverse of the diagonal elements of the Fisher information matrix. The RCRB along the $x$ and $y$-axis are equal and its expression is

$$
\mathrm{RCRB}=\left[N_{0} \sum_{i=-K}^{K} \sum_{j=-K}^{K} \frac{\left(\int_{\left(j-\frac{1}{2}\right) \Delta_{x y}}^{\left(j+\frac{1}{2}\right) \Delta_{x y}}\left[f^{\psi, \boldsymbol{\theta}_{0}}\left(\left(i-\frac{1}{2}\right) \Delta_{x y}, y\right)-f^{\psi, \boldsymbol{\theta}_{0}}\left(\left(i+\frac{1}{2}\right) \Delta_{x y}, y\right)\right] \mathrm{d} y\right)^{2}}{\int_{\left(i-\frac{1}{2}\right) \Delta_{x y}}^{\left(i+\frac{1}{2}\right) \Delta_{x y}} \int_{\left(j-\frac{1}{2}\right) \Delta_{x y}}^{\left(j+\frac{1}{2}\right) \Delta_{x y}} f^{\psi, \boldsymbol{\theta}_{0}}(x, y) \mathrm{d} x \mathrm{~d} y}\right]^{-\frac{1}{2}} .
$$

We note that the fundamental limit on localization is inversely proportional to the square root of the total number of photo-electrons $\sqrt{N_{0}}$ expected in the image and depends on the spatial sampling of the PSF.

In Fig. 2.b, we have represented the variation of RCRB as a function of defocus for an example of microscope configuration. Two $y$-axis are displayed: the left one uses an absolute nanometric unit in the object space, and the second one uses an arbitrary unit (a.u.) chosen such as $\operatorname{RCRB}(\psi=0)=1$. We have represented $\operatorname{RCRB}$ only over the positive values of $\psi$ since it is symmetric. It is observed that RCRB increases slowly until $\psi=\lambda / 4$, then the increase gets much sharper. The range $|\psi|<\lambda / 4$ corresponds to the Rayleigh criterion: it is classically considered that defocus is not critical for this range. It is noticed that for a defocus parameter of $\psi=1 \lambda$, RCRB is 2.9 times larger than for a focused particle (i.e. $\psi=0$ ).

Since we have chosen a signal-dependent photon noise model, the value of RCRB is inversely proportional to $\sqrt{N_{0}}$, as it appears in Eq. 7. Thus, the absolute value of the RCRB in Fig. 2.b depends on $N_{0}$, but the global shape of the curve does not. So we will adopt for the next plots the normalized RCRB.

\section{CO-DESIGN APPROACH}

As we just showed in the previous section, the lateral resolution in localization microscopy depends on the defocus. Our objective is to make the location accuracy of a single-molecule as invariant as possible to defocus. 

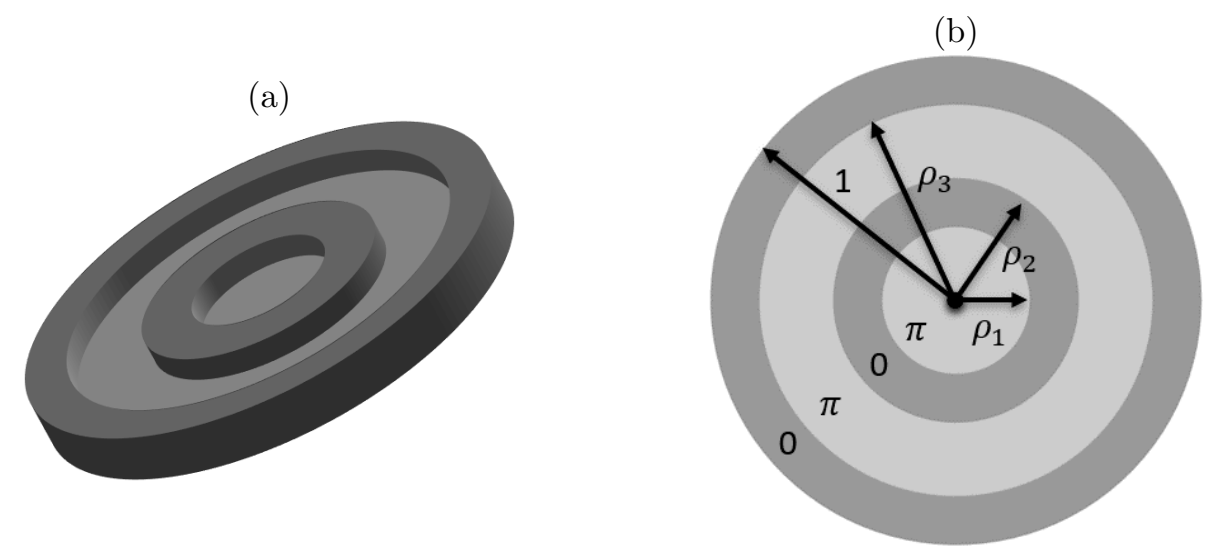

Figure 3. Example of four-ring annular binary phase mask (a) in 3D and (b) in 2D, defined by $\boldsymbol{\rho}=\left(\rho_{1}, \rho_{2}, \rho_{3}\right)^{\mathrm{t}}$. Each ring is defined as an annular region with constant phase modulation. Dark gray areas induce a phase of 0 radians and light gray areas induce a phase of $\pi$ radians at a nominal wavelength $\lambda$.

In other words, we want to extend the DoF of the localization microscope. We therefore propose to co-design annular binary phase masks in order to generate a PSF which achieves this goal.

\subsection{Annular binary phase masks}

Annular binary phase masks are static spatial phase modulating optical elements (see Fig. 3.a) defined by the normalized outer radius of their concentric rings (see Fig. 3.b), where each ring introduces a phase modulation of 0 or $\pi$ radians at a nominal wavelength $\lambda$. For example, we have represented in Figs. 3 a four-ring annular binary phase mask defined by the parameter vector of normalized radius $\boldsymbol{\rho}=\left(\rho_{1}, \rho_{2}, \rho_{3}\right)^{\mathrm{t}}$, with $0 \leq \rho_{1} \leq \rho_{2} \leq \rho_{3} \leq 1$, where $\rho_{n}$ is the outer normalized radius of the $n$th ring. The binary phase function defined by the mask is $\Phi_{\text {mask }}(r, \boldsymbol{\rho})$.

In consequence, the pupil phase function corresponding to an out-of-focus object, previously defined in Eq. (1), becomes

$$
\Phi(r, \boldsymbol{\rho})=\frac{2 \pi \psi r^{2}}{\lambda}+\Phi_{\operatorname{mask}}(r, \boldsymbol{\rho}), \quad r \in[0,1] .
$$

An interesting property of annular binary phase masks with $\pi$-phase modulation is that the PSF is symmetrical on either side of the focus point. ${ }^{20}$ So we continue to represent the plots for positive defocus values only.

\subsection{Co-design optimization criteria}

Co-optimization for DoF extension consists in optimizing optical parameters, here the mask parameters $\boldsymbol{\rho}$, in order to obtain a "good" estimated molecule position. But what does "good" mean? And how to perform this optimization?

A first reasonable criterion for phase mask optimization is to minimize the value of the fundamental limit of localization accuracy for the worst defocus parameter. ${ }^{18}$ In mathematical terms, the optimized binary phase mask parameters, called $\boldsymbol{\rho}_{\mathrm{opt}}$, are defined by

$$
\boldsymbol{\rho}_{\text {opt }}\left(\psi_{\text {max }}\right)=\arg \min _{\boldsymbol{\rho}}\left\{\max _{\psi \in\left[0, \psi_{\max }\right]} \operatorname{RCRB}(\boldsymbol{\rho}, \psi)\right\}
$$

where $\left[0, \psi_{\max }\right]$ characterize the defocus range on which we want localization to be accurate.

This minimax criterion makes it possible to ensure a certain localization accuracy by limiting the maximal RCRB value. However, for some applications, it might be preferable to optimize the average of the RCRB over the desired DoF range, rather than its maximal value. Thus, we propose to compare in the next section, the minimax criterion with a second co-design optimization criterion called minimean criterion and defined as

$$
\boldsymbol{\rho}_{\text {opt }}\left(\psi_{\text {max }}\right)=\arg \min _{\boldsymbol{\rho}}\left\{\operatorname{mean}_{\psi \in\left[0, \psi_{\text {max }}\right]} \operatorname{RCRB}(\boldsymbol{\rho}, \psi)\right\} .
$$




\section{ANNULAR BINARY PHASE MASK OPTIMIZATION}

By applying the optimization criteria defined in the previous section, we optimize annular binary phase masks for various defocus ranges. Since these optimization criteria are not simple and present several local minima, we start by restricting our attention to two-ring mask co-optimization, since this problem involves only a single parameter. We then consider co-optimization of masks with more than two rings.

\subsection{Two-ring phase masks}

Since a two-ring phase mask is defined by a single parameter $\boldsymbol{\rho}=\rho_{1}$, the optimization of this parameter can be easily performed by exhaustive search. Figure 4.a compares the two co-optimization criteria by plotting, as a function of the defocus range $\psi_{\max }$, the values of RCRB obtained with and without the optimal mask of parameter $\rho_{\text {opt }}$. The blue line corresponds to the maximal value of the RCRB over the defocus range, called $\mathrm{RCRB}_{\max }$, obtained with an aberration-free optical system without phase mask and can be used as a reference for comparison. The orange and purple areas correspond respectively to the set of RCRB values obtained for all values $\psi \in\left[0, \psi_{\max }\right]$, using a mask optimized with the minimean and the minimax criterion. Figure 4 .b gives the value of the optimal mask parameter $\rho_{\text {opt }}$ as a function of the defocus range.

The main conclusion that can be drawn from these graphs is that optimal binary masks significantly improve localization accuracy for all the defocus ranges such that $\psi_{\max }>0.3 \lambda$ whatever the optimization criterion used. For instance, when the defocus range is equal to $\psi_{\max }=0.7 \lambda$, the two-ring binary mask optimal for the minimax criterion yields a $\mathrm{RCRB}_{\max } 2$ times smaller than the one obtained without mask. For the mask optimal for the minimean criterion, it is 1.5 times smaller. For small defocus ranges such that $\psi_{\max }<0.3 \lambda$, the optimal mask parameter is not null but it leads to the same RCRB as no mask at all, so that binary phase masks do not improve localization accuracy for these ranges.

In Fig. 4.c, we have represented the evolution of the normalized RCRB as a function of defocus for an optical system using a two-ring binary phase mask optimized for $\psi_{\max }=0.7 \lambda$. We note that depending on the criterion used, the compromise on the values of RCRB is not the same. Indeed, the ratio between the RCRB obtained with minimax criterion and the RCRB obtained with the minimean criterion is equal to 1.32 for $\psi=0$ and to 0.81 for $\psi=\psi_{\max }=0.7 \lambda$. The minimean criterion promotes good localization accuracy for low DoF values, while the minimax criterion seeks to limit the maximum value of the RCRB over the defocus range. The choice of the optimization criterion is therefore an important component of the phase mask optimization problem and depends of the desired final application. We have presented in this section two criteria of co-optimization but of course, others are possible such as for example a criterion based on a linear combination of the RCRB obtained for different defocus parameters. In the next section, we will only focus on the minimax criterion.

\subsection{Multi-ring phase masks}

In this section, we address the following question: is it possible that binary phase masks with more rings, and therefore more degree of freedom in optimization, further improve localization accuracy? We will discuss the performance limits of multi-ring binary phase masks for depth-of-field extension in localization microscopy as it has been investigated for conventional imaging systems ${ }^{20}$ or for localization microscopy when the measurement noise is normally distributed. ${ }^{18}$

To answer this question, we optimize the minimax criterion defined in Eq. (9) with a larger number of rings. Figure 5.a represents the maximal value of the normalized RCRB over the defocus range obtained with the optimal mask parameter set $\boldsymbol{\rho}_{\mathrm{opt}}$, defined as

$$
\mathrm{RCRB}_{\max }=\max _{\psi \in\left[0, \psi_{\max }\right]} \operatorname{RCRB}\left(\boldsymbol{\rho}_{\mathrm{opt}}, \psi\right)
$$

This value is plotted as a function of the defocus range until $\psi_{\max }=2 \lambda$. The blue line is obtained with an optical system without mask. Similarly to Fig. 4.a, the RCRB increases slowly until $\psi=\lambda / 4$, then increases more rapidly, steps down from $\psi_{\max } \simeq 0.7 \lambda$, then increases again sharply from $\psi_{\max } \simeq 1 \lambda$, then has a series of successive steps until $\psi_{\max }=2 \lambda$. These steps reflect the fact that an increase in $\psi_{\max }$ may not always lead to an increase of $\mathrm{RCRB}_{\max }$. The red line is obtained using a two-ring mask optimized for the corresponding defocus 
(a)

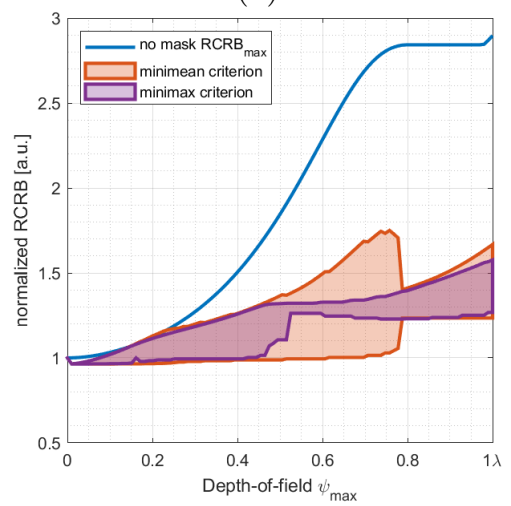

(b)

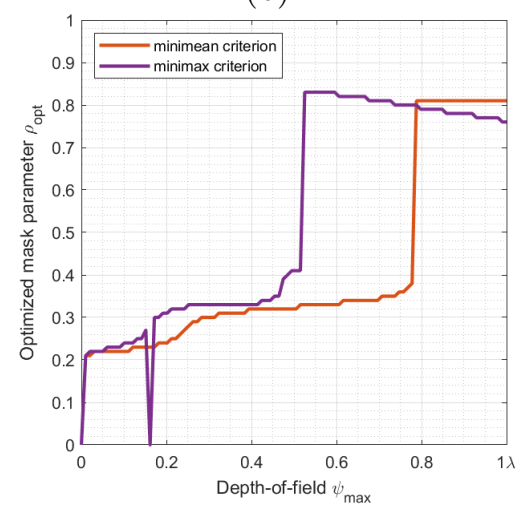

(c)

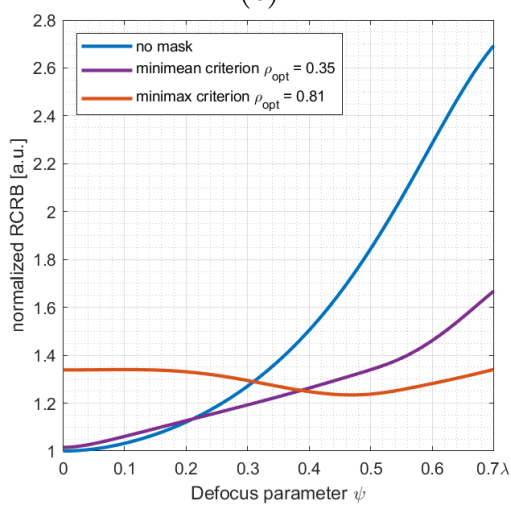

Figure 4. (a) Evolution of the maximum $\left(\mathrm{RCRB}_{\max }\right)$ and minimum $\left(\mathrm{RCRB}_{\min }\right)$ of the RCRB as a function of the defocus range, characterized by $\psi_{\max }$, of a localization microscope using a two-ring binary phase mask. (b) Evolution of the optimized parameter mask $\rho_{\text {opt }}$ as a function of the defocus range. (c) Evolution of RCRB as a function of defocus for an optical system using a binary phase mask optimized for $\psi_{\max }=0.7 \lambda$. Simulation parameters: $\boldsymbol{\theta}_{0}=(0,0)^{\mathrm{t}} \mu \mathrm{m}, N_{0}=500$ photo-electrons, $\Delta_{x y}=10 \mu \mathrm{m}, 2 K+1=21$ pixels, $\mathrm{NA}=1.3$ (object numerical aperture), $\lambda=700 \mathrm{~nm}$ (wavelength), and $M=60$ (magnification).

range. As pointed out in the previous section, for $\psi_{\max }>0.3 \lambda$, the use of such a phase mask makes it possible to reduce the $\mathrm{RCRB}_{\max }$ and therefore increases the DoF of the optical system. For instance, for $\psi_{\max }=2 \lambda$ the use of an optimized two-ring binary phase mask reduces by half the $\mathrm{RCRB}_{\max }$. The curve with yellow dots is obtained using an optimized multi-ring binary phase mask. Since it is no longer possible to use an exhaustive search to achieve the multi-ring non-convex optimization defined in Eq. (9) with a larger number of rings, we use the particle swarm optimization algorithm. We performed this phase mask optimization for discrete values of $\psi_{\max }$ spaced by $0.1 \lambda$ from 0 to $2 \lambda$. We can see that using a multi-ring mask further reduces the $\mathrm{RCRB}_{\max }$. In other words, these additional degrees of freedom in the optimization lead to obtain more efficient phase masks. For example, for $\psi_{\max }=2 \lambda$, the use of an optimized multi-ring binary phase mask makes it possible to divide by 3 the $\mathrm{RCRB}_{\max }$. However we note again that for $\psi_{\max }<0.3 \lambda$, phase masks, whatever their number of rings, are useless since the obtained performance is identical to that obtained without mask.

Figure 5.b represents the parameters of the optimal multi-ring masks whose localization performance is given by the curve with yellow dots in Fig. 5.a. The outer radii of the optimized annular mask rings are displayed as a function of the defocus range $\psi_{\max }$. As highlighted previously, the additional degrees of freedom in the optimization make it possible to obtain more efficient phase masks. In fact, we notice that the number of rings of the optimal mask increases with the defocus range $\psi_{\max }$. Indeed, from $\psi_{\max }=0$ to $0.4 \lambda$ the optimal annular binary phase masks have two rings, from $\psi_{\max }=0.4 \lambda$ to $0.8 \lambda$ three rings, from $\psi_{\max }=0.8 \lambda$ to $1.2 \lambda$ four rings, and for $\psi_{\max }>1.2 \lambda$ at least four rings. Similar results have already been obtained in classical imaging systems where the DoF is improved by placing a binary phase mask in the pupil of the objective and by restoring the image with an appropriate deconvolution filter system. ${ }^{20}$ In addition, it is seen in Fig. 5.b that the radii of the rings of the optimal masks vary almost continuously with the defocus range, and "split" into two values when one more ring is necessary. The few discontinuities, illustrated by dotted curves, correspond to different local minima in the optimization problem.

\section{CONCLUSION}

We have investigated the problem of DoF extension in localization microscopy with a Poissonian noise model (see our previous work ${ }^{18}$ for more details in the Gaussian case) in order to answer our initial question: Can phase masks extend depth-of-field in localization microscopy? This work is different from that presented in our previous work $^{18}$ where the measurement noise is normally distributed.

We have shown that placing an optimized multi-ring annular binary phase mask in the exit pupil of the localization microscope allows to significantly increase the localization performance within the required defocus 
(a)

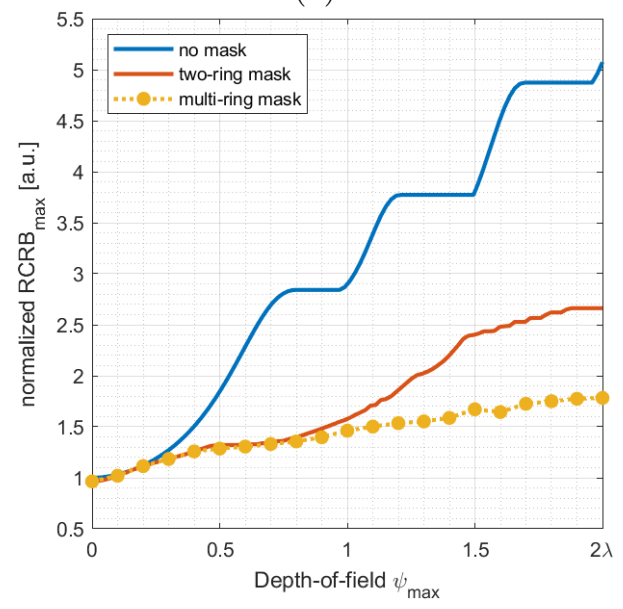

(b)

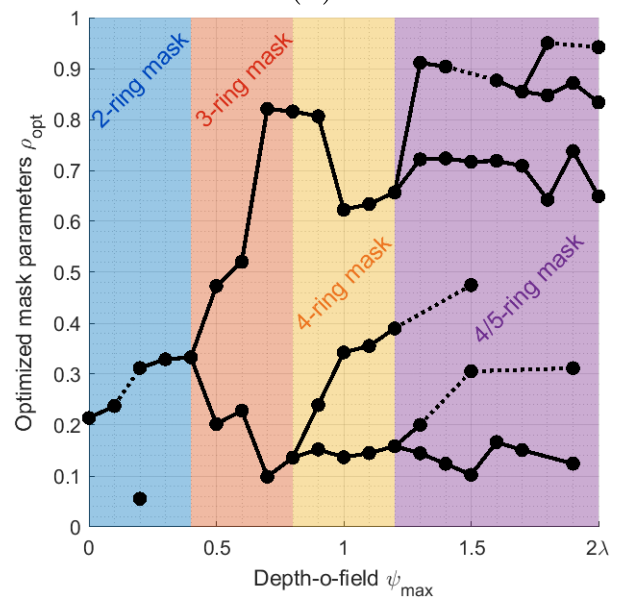

Figure 5. (a) Evolution of the $\mathrm{RCRB}_{\max }$ as a function of the defocus range, characterized by $\psi_{\max }$, of a localization microscope using a multi-ring binary phase mask. (b) Evolution of the optimized parameter mask $\boldsymbol{\rho}_{\mathrm{opt}}$ as a function of the defocus range $\psi_{\text {max }}$. Simulation parameters: $\boldsymbol{\theta}_{0}=(0,0)^{\mathrm{t}} \mu \mathrm{m}, N_{0}=500$ photo-electrons, $\Delta_{x y}=10 \mu \mathrm{m}, 2 K+1=21$ pixels, NA $=1.3$ (object numerical aperture), $\lambda=700 \mathrm{~nm}$ (wavelength), and $M=60$ (magnification).

range. For example for a defocus range of $\psi_{\max }=2 \lambda$, the optimized annular binary phase mask reduces the RCRB by a factor 3 compared to a standard system. Additionally, we also noticed that the larger the desired DoF extension, the more rings the optimal mask needs.

The framework developed in this article makes it possible to optimize and compare the performance of any type of DoF-enhancing mask, since it is based on an explicit and general information theoretical criterion. The presented results take into account a signal-dependent photon noise model. An interesting perspective of this work is to extend this approach to other mask models, and to compare their performance in terms of DoF extension.

Another perspective is to use a more accurate theoretical description of the PSF of optical localization microscopes. The present work demonstrated the utility of the annular binary phase mask for the DoF extension problem in localization microscopy using a simple imaging model taking into account signal-dependent photon noise and defocus aberration. However, more accurate theoretical description of the PSF exist in the literature ${ }^{21}$ and could be implemented within this framework.

\section{REFERENCES}

[1] Hess, S. T., Girirajan, T. P., and Mason, M. D., "'Ultra-High Resolution Imaging by Fluorescence Photoactivation Localization Microscopy"," Biophysical Journal 91(11), 4258 - 4272 (2006).

[2] Betzig, E., Patterson, G. H., Sougrat, R., Lindwasser, O. W., Olenych, S., Bonifacino, J. S., Davidson, M. W., Lippincott-Schwartz, J., and Hess, H. F., "Imaging Intracellular Fluorescent Proteins at Nanometer Resolution," Science 313(5793), 1642-1645 (2006).

[3] Chao, J., Ward, E. S., and Ober, R. J., "Fisher information theory for parameter estimation in single molecule microscopy: tutorial," J. Opt. Soc. Am. A 33, B36-B57 (Jul 2016).

[4] Ram, S., Sally Ward, E., and Ober, R. J., "A Stochastic Analysis of Performance Limits for Optical Microscopes," Multidimensional Systems and Signal Processing 17(1), 27-57 (2006).

[5] Ober, R. J., Ram, S., and Ward, E. S., "'Localization Accuracy in Single-Molecule Microscopy"," Biophysical Journal 86(2), 1185 - 1200 (2004).

[6] Hajj, B., El Beheiry, M., Izeddin, I., Darzacq, X., and Dahan, M., "'Accessing the third dimension in localization-based super-resolution microscopy"," Phys. Chem. Chem. Phys. 16, 16340-16348 (2014).

[7] von Diezmann, A., Shechtman, Y., and Moerner, W. E., "Three-Dimensional Localization of Single Molecules for Super-Resolution Imaging and Single-Particle Tracking," Chemical Reviews 117, 7244-7275 (Jun 2017). 
[8] Zahreddine, R. N., Cormack, R. H., and Cogswell, C. J., "Simultaneous quantitative depth mapping and extended depth of field for 4D microscopy through PSF engineering," in [Three-Dimensional and Multidimensional Microscopy: Image Acquisition and Processing XIX], Conchello, J.-A., Cogswell, C. J., Wilson, T., and Brown, T. G., eds., 8227, 26 - 32, International Society for Optics and Photonics, SPIE (2012).

[9] Pavani, S. R. P. and Piestun, R., "High-efficiency rotating point spread functions," Opt. Express 16, 34843489 (Mar 2008).

[10] Dowski, E. R., Cormack, R. H., and Sarama, S. D., "Wavefront coding: jointly optimized optical and digital imaging systems," in [Visual Information Processing IX], Park, S. K. and ur Rahman, Z., eds., 4041, 114 - 120, International Society for Optics and Photonics, SPIE (2000).

[11] Harvey, A. R., Vettenburg, T., Demenikov, M., Lucotte, B., Muyo, G., Wood, A., Bustin, N., Singh, A., and Findlay, E., "Digital image processing as an integral component of optical design," in [Novel Optical Systems Design and Optimization XI], Koshel, R. J., Gregory, G. G., Jr., J. D. M., and Krevor, D. H., eds., 7061, 32 - 42, International Society for Optics and Photonics, SPIE (2008).

[12] Diaz, F., Goudail, F., Loiseaux, B., and Huignard, J.-P., "Comparison of depth-of-focus-enhancing pupil masks based on a signal-to-noise-ratio criterion after deconvolution," J. Opt. Soc. Am. A 27, 2123-2131 (Oct 2010).

[13] Diaz, F., Goudail, F., Loiseaux, B., and Huignard, J.-P., "Increase in depth of field taking into account deconvolution by optimization of pupil mask," Opt. Lett. 34, 2970-2972 (Oct 2009).

[14] Sitzmann, V., Diamond, S., Peng, Y., Dun, X., Boyd, S., Heidrich, W., Heide, F., and Wetzstein, G., "End-to-End Optimization of Optics and Image Processing for Achromatic Extended Depth of Field and Super-Resolution Imaging," ACM Trans. Graph. 37 (July 2018).

[15] Elmalem, S., Giryes, R., and Marom, E., "Learned phase coded aperture for the benefit of depth of field extension," Opt. Express 26, 15316-15331 (Jun 2018).

[16] Cathey, W. T. and Dowski, E. R., "New paradigm for imaging systems," Appl. Opt. 41, 6080-6092 (Oct 2002).

[17] Robinson, M. D. and Stork, D. G., "Joint Design of Lens Systems and Digital Image Processing," in [International Optical Design], International Optical Design, WB4, Optical Society of America (2006).

[18] Lévêque, O., Kulcsár, C., Lee, A., Sauer, H., Aleksanyan, A., Cognet, L., Bon, P., and Goudail, F., "Codesigned annular binary phase masks for depth-of-field extension in single-molecule localization microscopy," to appear in Opt. Express (2020).

[19] Born, M., Wolf, E., Bhatia, A. B., Clemmow, P. C., Gabor, D., Stokes, A. R., Taylor, A. M., Wayman, P. A., and Wilcock, W. L., [Principles of Optics: Electromagnetic Theory of Propagation, Interference and Diffraction of Light], Cambridge University Press, 7th ed. (1999).

[20] Falcón, R., Goudail, F., Kulcsár, C., and Sauer, H., "Performance limits of binary annular phase masks codesigned for depth-of-field extension," Optical Engineering 56(6), 1 - 12 (2017).

[21] Aguet, F., Super-Resolution Fluorescence Microscopy Based on Physical Models, theses, École polytechnique fédérale de Lausanne (May 2009). 